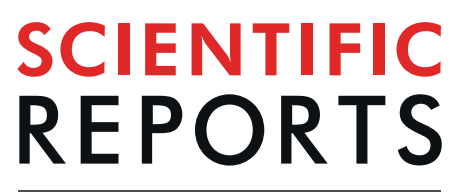

natureresearch

Check for updates

\title{
OPEN The effect of 17\% EDTA and QMiX ultrasonic activation on smear layer removal and sealer penetration: ex vivo study
}

\author{
Felipe de Souza Matos $\mathbb{1}^{1 凶}$, Fabrício Rutz da Silva $^{2}$, Luiz Renato Paranhos ${ }^{3}$, \\ Camilla Christian Gomes Moura ${ }^{4}$, Eduardo Bresciani ${ }^{5}$ \& Marcia Carneiro Valera ${ }^{5}$
}

This study aimed to compare the effect of conventional irrigation $(\mathrm{CI})$ and passive ultrasonic irrigation (PUI) with 17\% EDTA and QMiX on the maximum depth and percentage of sealer penetration into the dentinal tubules by confocal laser scanning microscopy (CLSM) and to describe the cleaning of root canal walls by scanning electron microscopy (SEM). Eighty single-rooted human mandibular premolars were instrumented and randomly assigned to four groups $(n=20)$ : EDTA $+\mathrm{Cl}, \mathrm{QMiX}+\mathrm{Cl}, \mathrm{EDTA}+\mathrm{PUI}$, and $\mathrm{QMiX}+\mathrm{PUI}$. Ten samples from each group were examined by SEM $(2,000 \times)$ and the remaining 40 roots were filled with a single gutta-percha cone and $A H$ Plus sealer mixed with $0.1 \%$ rhodamine $B$ for analysis by CLSM $(10 \times)$. Images were assessed at distances of $2 \mathrm{~mm}$ (apical), $5 \mathrm{~mm}$ (middle), and $8 \mathrm{~mm}$ (coronal) from the apex with the Leica Application Suite V4.10 software. The EDTA + PUI and QMiX + PUI protocols presented higher rates of debris/smear layer removal in the apical and middle thirds. The $\mathrm{PUI}$ was superior to $\mathrm{Cl}$ in the maximum depth of sealer penetration at the middle third. The $\mathrm{QMiX}+\mathrm{PUI}$ group had a higher percentage of sealer penetration at the apical third. The PUI and QMiX protocol improved debris/smear layer removal and tubular dentin sealer penetration.

The success of endodontic therapy depends on the chemomechanical disinfection and appropriate antimicrobial sealing of the root canal system (RCS) ${ }^{1}$. The biomechanical preparation using mechanical instrumentation and antimicrobial solutions aims to shape the root canal and either eliminate or reduce toxic and necrotic contents, including pulp remains and pathogens ${ }^{2}$. However, as a result of instrumentation, a 1- to 2- $\mu \mathrm{m}$ thick smear layer primarily composed of inorganic dentin is formed in the root canal walls and it should be removed during the final irrigation with chelating agents because it blocks the dentinal tubules, harboring necrotic debris and bacteria and their by-products ${ }^{3}$. It also limits the penetration of disinfectants and sealers into the dentinal tubules ${ }^{4}$.

Sodium hypochlorite $(\mathrm{NaOCl})$ is the main irrigating solution used in the endodontic treatment due to its antimicrobial action and solvent capacity on organic tissues, but it does not affect the inorganic content. To remove debris and smear layer and improve the permeability of the RCS, final irrigation with ethylenediaminetetraacetic acid (EDTA) is recommended as a prerequisite for a satisfactory sealing of the dentinal canaliculi ${ }^{5}$. More recently, the final irrigant QMiX has gained special attention for presenting a chelating effect similar or superior to EDTA, besides antimicrobial activity ${ }^{6-9}$. The QMiX promotes a superior sealer penetration to that achieved by other chelating solutions such as BioPure MTAD ${ }^{10}$.

Although the antimicrobial and chelating actions of endodontic irrigants play a critical role in disinfecting and cleaning the RCS, conventional needle irrigation may not allow these substances to work deep into the dentinal tubules. Thus, different devices and irrigant activation techniques have been developed and recommended to improve the efficiency and distribution of solutions ${ }^{11-13}$. Passive ultrasonic irrigation (PUI) activates the irrigant solution by acoustic microstreaming transmitted from an oscillating file or smooth wire at an ultrasonic

${ }^{1}$ Postgraduate Program in Dentistry, School of Dentistry, Federal University of Uberlândia (UFU), Uberlândia, MG, Brazil. ${ }^{2}$ Department of Restorative Dentistry, School of Dentistry, State University of Ponta Grossa (UEPG), Ponta Grossa, PR, Brazil. ${ }^{3}$ Department of Preventive and Social Dentistry, School of Dentistry, Federal University of

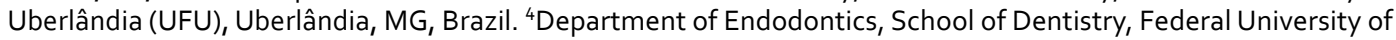
Uberlândia (UFU), Uberlândia, MG, Brazil. ${ }^{5}$ Department of Restorative Dentistry, Institute of Science and Technology,

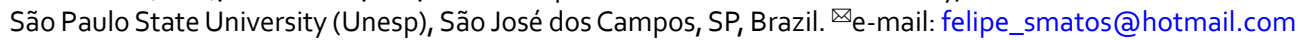


frequency of $30 \mathrm{kHz}$. It also improves the cleaning and disinfection of the RCS when compared to conventional needle irrigation ${ }^{11,14}$.

Several studies have shown that the proper filling of the RCS depends on the chelating capacity of chemicals used mainly during the final cleaning and on the concomitant use of some activation systems ${ }^{15-17}$. Although some studies report that QMiX and 17\% EDTA exhibit similar root canal cleaning ability ${ }^{6}$, the effect of different activation techniques of these substances on smear layer removal and sealer penetration has not yet been well explained $^{11,17,18}$. The penetration of sealer into the dentinal tubules is clinically important because adequate sealing may control infections and prevent recontamination ${ }^{19}$.

The effect of the combined use of QMiX and PUI on both the cleaning of root canal walls and sealer penetration into the dentinal tubules has not been studied to date. Therefore, the present study was conducted to compare the effect of conventional irrigation (CI) and passive ultrasonic irrigation (PUI) with 17\% EDTA and QMiX on the maximum depth and percentage of sealer penetration into the dentinal tubules by confocal laser scanning microscopy (CLSM). It also aims to describe the cleaning of root canal walls by scanning electron microscopy (SEM) at different root canal levels (cervical, middle, and apical thirds). The authors tested the following hypotheses: (1) There is no difference in the rate of debris/smear layer removal between conventional or passive ultrasonic irrigations with 17\% EDTA and QMiX; (2) There is no difference in the rate of tubular dentin sealer penetration between conventional or passive ultrasonic irrigations with 17\% EDTA and QMiX.

\section{Material and Methods}

Sample size. This study was approved by the Research Ethics Committee of the Institute of Science and Technology - Unesp (Certificate of Presentation for Ethical Consideration: 79730317.2.0000.0077) and the methods were carried out in accordance with the Declaration of Helsinki (2008). Sample size calculation was performed based on previous data ${ }^{10}$ with the $\mathrm{G}^{*}$ Power software (version 3.1), using the following parameters: two-tailed $5 \%$ significance level $(\alpha=0.05), 95 \%$ confidence interval, $90 \%$ statistical power $(\beta=0.10), 1: 1$ ratio of specimen allocation in the experimental groups, and medium estimated effect size $(\mathrm{d}=0.60)$, which indicated the need to include a minimum of 20 specimens in each group. Thus, a final study sample of 80 human mandibular premolars freshly extracted for orthodontic or periodontal reasons were used. The teeth were collected from the Department of Surgery, Periodontics and Radiology of the Institute of Science and Technology - Unesp, and all patients have obtained the informed consent.

Sample selection. The teeth were immersed in $0.1 \%$ thymol solution during 48 hours for disinfection and stored in distilled water at $4{ }^{\circ} \mathrm{C}$ until use. All the teeth were evaluated radiographically to confirm the presence of a single root canal, mature apex, and absence of any resorption or endodontic treatment. The study included only teeth with radicular canal widths of 3-4 mm buccolingually and 1-2 mm mesiodistally at the cementoenamel junction level, anatomic diameter of the \#15 or \#20 Kerr file (Dentsply Maillefer, Ballaigues, Switzerland) at $1 \mathrm{~mm}$ from the apex, and straight or curved canal up to $10^{\circ 20}$. The teeth selected were cross-sectioned in their long axes below the cementoenamel junction for removing the crown and standardizing the total root length at $12.0 \pm 0.5 \mathrm{~mm}$, using a diamond disc (Horico Dental Hopf, Ringleb \& Co GmbH \& Cie, Berlin, Germany).

Root canal preparation. The full length of the root canals was instrumented up to a \#30 Kerr file (Dentsply Maillefer, Ballaigues, Switzerland) using $3 \mathrm{~mL}$ of saline solution after each instrumentation to standardize the apical diameter. The canals were filled with 17\% EDTA (Inodon, Porto Alegre, RS, Brazil) for three minutes and irrigated with $10 \mathrm{~mL}$ of saline solution. Next, the apical foramen was sealed with light-cured composite resin (Z-100, 3 M, Saint Paul, USA) to create an apical seal. The biomechanical instrumentation was performed with the R40 WaveOne single-file reciprocating system (Dentsply Maillefer, Ballaigues, Switzerland) according to the crown-down technique (i.e., coronal, medium, and apical) associated with irrigation of $5 \mathrm{~mL}$ of $2.5 \%$ sodium hypochlorite (NaOCl) (Fórmula e Ação, São Paulo, Brazil) for each third, totaling $15 \mathrm{~mL}$ for each sample. The apical working length (WL) was established at $1 \mathrm{~mm}$ short of the anatomical apex and irrigation was performed using a 5-mL disposable syringe (Ultradent, South Jordan, USA) and a 30-gauge NaviTip needle (Ultradent, South Jordan, USA) inserted into the canal $2 \mathrm{~mm}$ short of the WL. Foraminal patency was maintained with a \#15 Kerr file. The root canals were rinsed with $10 \mathrm{~mL}$ of saline solution to neutralize $\mathrm{NaOCl}$.

Final irrigation. After instrumentation, the samples were randomly assigned to four groups $(\mathrm{n}=20$ each) according to the final irrigation protocol used: conventional irrigation (CI) or passive ultrasonic irrigation (PUI) with $17 \%$ EDTA and QMiX, which are described below.

EDTA + CI. The samples were flooded with 17\% EDTA solution (Inodon, Porto Alegre, RS, Brazil) for two minutes without any type of agitation.

$\mathrm{QMiX}+\mathrm{CI}$. The samples were flooded with QMiX (Dentsply Tulsa Dental Specialties, Johnson City, TN, USA) for two minutes without any type of agitation.

EDTA + PUI. The samples were flooded with 17\% EDTA solution (Inodon, Porto Alegre, RS, Brazil) for two minutes and ultrasonically agitated in the last 60 seconds.

$\mathrm{QMiX}+$ PUI. The samples were flooded with QMiX (Dentsply Tulsa Dental Specialties, Johnson City, TN, USA) for two minutes and ultrasonically agitated in the last 60 seconds.

All root canals were irrigated with $3 \mathrm{~mL}$ of the respective final irrigating solution using a 5 -mL disposable syringe and a 30 -gauge NaviTip needle placed into the canal $2 \mathrm{~mm}$ short of the WL. The ultrasonic activation was performed at a minimum power setting (10\%), using an Irrisonic tip (Helse, Santa Rosa do Viterbo, SP, Brazil) inserted into the canal $1 \mathrm{~mm}$ short of the WL. After removing the excess of the final irrigant, the canals were rinsed with $10 \mathrm{~mL}$ of saline solution and dried with paper points. 

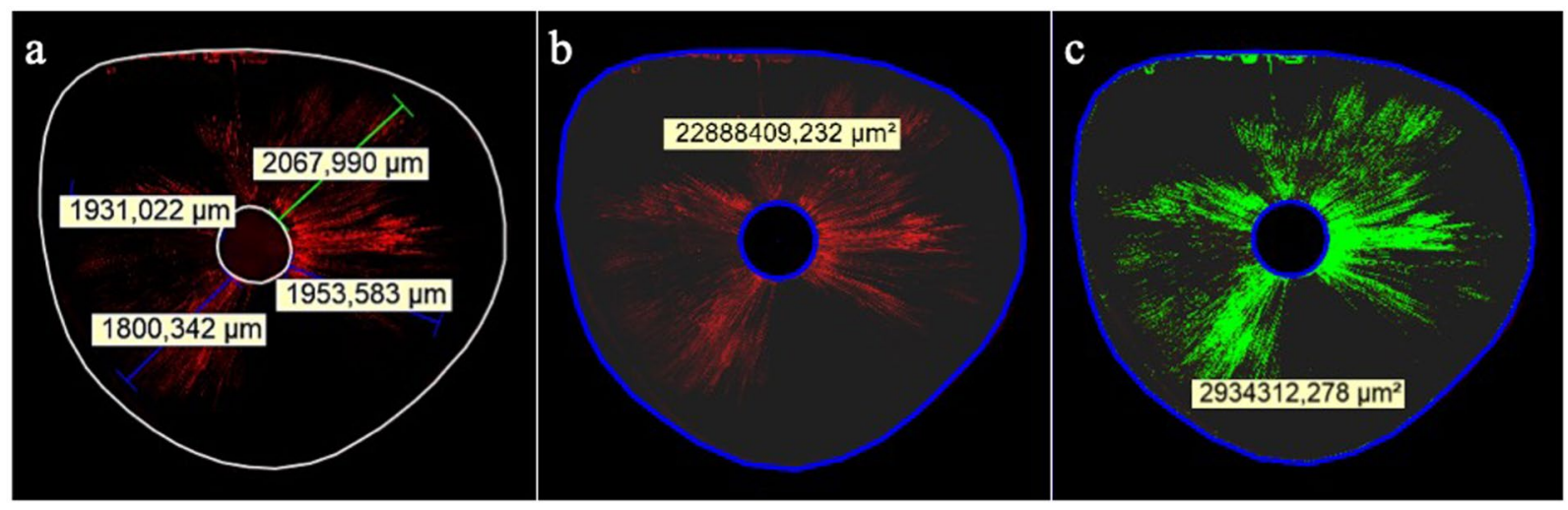

Figure 1. Maximum depth and percentage of sealer penetration: (a) Four measurements of the penetration depth were recorded in the slice and maximum penetration was considered (green line); (b) The total area of the canal wall was recorded; and (c) Areas of sealer penetration were measured (green areas).

Scanning electron microscopy evaluation. To describe the effect of the final irrigation protocol on the removal of debris and smear layer from the root canal walls, 10 samples from each group were prepared for scanning electron microscopy (SEM). Two longitudinal grooves were made along the external root surface in the mesiodistal direction using a diamond disc, and the samples were split in half with a chisel to expose the root canal. The root halves were dehydrated in ascending ethanol and dried in an incubator at $37^{\circ} \mathrm{C}$ for 24 hours. Subsequently, they were mounted on stubs and coated with 20-nm thick gold-palladium for the SEM evaluation. One representative image of the apical $(2-3 \mathrm{~mm})$, middle $(5-4 \mathrm{~mm})$, and coronal $(8-9 \mathrm{~mm})$ thirds from each sample were obtained at a magnification of 2,000×, totaling 120 images. The efficacy of debris and smear layer removal was evaluated blindly by two investigators using a four-level scoring system: $0=$ no debris/smear layer with all tubules open; $1=$ minimum quantity of debris/smear layer with over $50 \%$ of the tubules open; $2=$ moderate quantity of debris/smear layer with less than $50 \%$ of the tubules open; $3=$ heavy debris/smear layer with almost all dentin tubules obstructed ${ }^{21}$. The investigators were previously calibrated for the scoring system, which they applied to a sample of $20 \%$ of the SEM images randomly selected from two specimens of each group $(n=24)$ to determine the inter-examiner agreement. After achieving a proper level of agreement (Kappa $\geq 0.81$ ), the investigators scored the images independently. The lowest score was chosen when there were conflicting results among the investigators. The scores were statistically evaluated using the Kruskal-Wallis test followed by Dunn's test, at $5 \%$ significance level.

Root canal filling. The remaining 40 roots were filled with AH Plus sealer (Dentsply; DeTrey, Konstanz, Germany) and a single R40 WaveOne gutta-percha cone (Dentsply Maillefer, Ballaigues, Switzerland) according to their manufacturers' instructions. The sealer was mixed with $0.1 \%$ fluorescent rhodamine B isothiocyanate dye (Sigma-Aldrich, St Louis, MO, USA) to allow the analysis under confocal microscope and it was placed into the canal using a \#25.02 Lentulo spiral (Dentsply Maillefer, Ballaigues, Switzerland) attached to a handpiece at $20,000 \mathrm{rpm}$, inserted into the canal $1 \mathrm{~mm}$ short of the WL for five seconds. The excess of gutta-percha was removed with a heated Schilder ${ }^{\mathrm{TM}}$ plugger (Dentsply Maillefer, Ballaigues, Switzerland) and vertical compaction was performed at the orifice level. The coronal access was sealed with light-cured composite resin and the samples were stored in an incubator at $37^{\circ} \mathrm{C}$ and $100 \%$ humidity for seven days to allow the sealer to set completely ${ }^{10}$.

Confocal laser scanning microscope evaluation. Each sample was sectioned perpendicular to its long axis in three 1-mm thick slices using a diamond blade in IsoMet (Buehler, Illinois, USA) at distances of $2 \mathrm{~mm}$ (apical), $5 \mathrm{~mm}$ (middle), and $8 \mathrm{~mm}$ (coronal) from the apex. The coronal surfaces of the slices were polished using silicon carbide abrasive papers to eliminate dentin debris produced during the cutting procedure. Then, they were mounted onto glass slides and examined under a Leica TCS SP 5 confocal laser scanning microscope (Leica Microsystems, Mannheim, Germany) at a magnification of $10 \times$ and wavelength of 540-590 nm. The Leica Application Suite V4.10 software (Leica Microsystems Ltd., Heerbrugg, Switzerland) was used to measure the maximum depth and percentage of sealer penetration into the dentinal tubules with a calibrated measuring tool (Fig. 1). The maximum depth of sealer penetration was measured from the root canal wall to the point of deepest penetration in the dentinal tubules (Fig. 1a). Areas along the root canal walls in which the sealer had penetrated were measured and divided by the total area along the canal walls to determine the percentage of sealer penetration (Fig. 1b,c). The means of percentage and maximum depth of sealer penetration for each root canal level were evaluated using ANOVA followed by Tukey's test, at $5 \%$ significance level.

\section{Results}

Scanning electron microscopy evaluation. The Kappa test showed an almost perfect level of agreement between the investigators (Kappa $=0.923$ ). Table 1 summarizes the results of the evaluation of debris and smear layer removal. Lower scores for debris/smear layer removal were achieved by the EDTA + PUI and QMiX + PUI groups in the apical and middle thirds when compared with the EDTA $+\mathrm{CI}$ and QMiX + CI groups $(\mathrm{p}<0.05)$. There were no statistically significant differences among the protocols in the coronal third $(p>0.05)$. In the 


\begin{tabular}{|l|l|l|l|l|}
\hline Groups & Apical third & Middle third & Coronal third & $\boldsymbol{p}$ value \\
\hline EDTA + CI & $3(3 ; 3)^{\mathrm{aA}}$ & $2(1 ; 2)^{\mathrm{aB}}$ & $1(0 ; 1)^{\mathrm{aC}}$ & $<0.001$ \\
\hline QMiX + CI & $3(3 ; 3)^{\mathrm{aA}}$ & $2(1 ; 2)^{\mathrm{aB}}$ & $1(0 ; 1)^{\mathrm{aC}}$ & $<0.001$ \\
\hline EDTA + PUI & $2(2 ; 3)^{\mathrm{bA}}$ & $1(1 ; 1)^{\mathrm{bB}}$ & $1(0 ; 1)^{\mathrm{aB}}$ & $<0.001$ \\
\hline QMiX + PUI & $2(2 ; 3)^{\mathrm{bA}}$ & $1(0 ; 1)^{\mathrm{bB}}$ & $1(0 ; 1)^{\mathrm{aB}}$ & $<0.001$ \\
\hline$p$ value & $<0.05$ & $<0.05$ & $>0.05$ & \\
\hline
\end{tabular}

Table 1. Comparison among groups considering the scores of debris/smear layer removal (median and IQR) in each root canal third. Dunn's test (multiple comparisons): different lowercase letters represent statistically significant difference among groups $(\mathrm{p}<0.05)$ and different capital letters represent statistically significant difference among thirds $(\mathrm{p}<0.05)$. IQR $=$ interquartile range.

EDTA + CI and QMiX + CI groups, the greatest debris/smear layer removal was observed in the coronal third, followed by the middle and apical thirds, respectively $(\mathrm{p}<0.001)($ Table 1$)$. In the EDTA + PUI and QMiX + PUI groups, a higher debris/smear layer removal was obtained in the coronal and middle thirds than in the apical third $(\mathrm{p}<0.001)$ (Table 1). Figure 2 shows representative SEM images of debris and smear layer removal from the root canal walls in each root third after the final irrigation protocols.

Confocal laser scanning microscope evaluation. Tables 2 and 3 present the means and standard deviations of maximum depth and percentage of sealer penetration at each root canal third, respectively. There was no statistically significant difference in the maximum depth of sealer penetration among the final irrigation protocols in the apical and coronal thirds $(\mathrm{p}>0.05)$. There was a statistically significant difference in the middle third between PUI and CI for both final irrigating solutions, with better results for the EDTA + PUI and QMiX + PUI groups $(\mathrm{p}<0.001)$. In all groups, the greatest depth of sealer penetration was observed in the coronal third, followed by the middle and apical thirds, respectively $(\mathrm{p}<0.001)$ (Table 2$)$. Figure 3 shows representative patterns of sealer penetration around the root canal walls in each group and root third.

Regarding the percentage of sealer penetration, there was no statistically significant difference among the final irrigation protocols only in the middle third $(\mathrm{p}>0.05)$. At the apical third, QMiX + PUI presented a significantly higher percentage of sealer penetration than the other groups $(p<0.001)$, followed by EDTA + PUI and conventional irrigation with EDTA or QMiX, with no statistical difference between EDTA + CI and QMiX + CI $(\mathrm{p}>0.05)$. The groups showed similar percentages of sealer penetration in the coronal third, but QMiX $+\mathrm{CI}$ performed better than EDTA $+\mathrm{CI}(\mathrm{p}<0.05)$. As in the previous analysis, the coronal third presented the highest percentage values of sealer penetration around the root canal walls, regardless of the final irrigation protocol, followed by the middle and apical thirds, respectively $(\mathrm{p}<0.001)$. However, in the QMiX + PUI group, the apical and middle thirds presented statistically similar percentages of sealer penetration (Table 3 and Fig. 3 ).

\section{Discussion}

The quality of root canal cleaning and filling is a significant predictor of successful endodontic treatment ${ }^{1}$. According to Chugal et al., a poor root filling may increase about twice the rate of treatment failure of teeth without periapical lesion, and this rate is $20 \%$ higher for teeth with apical periodontitis ${ }^{1}$. The presence of smear layer contributes not only to the ineffective obturation ${ }^{16}$ but also to the adhesion and colonization of bacteria and endotoxins in the dentin matrix ${ }^{22}$. The sealer penetration into the dentinal tubules is desirable because it might bury residual microorganisms and their toxins, keeping them away from nutrient sources and preventing reinfection ${ }^{23}$. Thus, considering the influence of the quality of final cleaning and obturation on the endodontic outcome, this study evaluated the effect of conventional irrigation (CI) or passive ultrasonic irrigation (PUI) with 17\% EDTA and QMiX on debris/smear layer removal and tubular dentin sealer penetration in ex vivo human root canals. Hypothesis (1) and (2) were rejected, as the final irrigation protocols tested differ in their effects on debris/smear layer removal and tubular dentin sealer penetration.

The results of this study showed that PUI with both 17\% EDTA and QMiX final irrigants improved the removal of debris/smear layer in the middle and apical thirds of the root canal when compared to CI. These findings are consistent with other studies that observed a greater smear layer removal when supplementing the final irrigation with PUI ${ }^{11,18,24-26}$. The smear layer is an amorphous structure containing mostly inorganic dentin debris and organic substances such as fragments of the odontoblastic process, microorganisms, and necrotic pulp tissue $e^{4}$. Ultrasonic activation potentially improved the debris/smear layer removal by causing shear stress in the inorganic particles of the smear layer by acoustic streaming, facilitating its removal ${ }^{14}$. However, the irrigant delivered by conventional needle only penetrates from 0 to $1.1 \mathrm{~mm}$ deeper than the tip of the needle, and gas particles are produced and trapped in the apical portion, creating a vapor lock and hindering the efficacy of irrigant debridement $^{12}$. On the other hand, PUI allows the elimination of vapor lock effect, improving the efficiency of the irrigating solution ${ }^{25}$. The safety of CI has also been questioned because the positive pressure used to deliver the solution into the canal may extrude it to the periapex, causing tissue damage and postoperative pain ${ }^{27}$.

The mechanism of action of chelating solutions is based on their ability to react with calcium ions in dentin and to form soluble calcium chelates ${ }^{4}$. The 2 -minute working time adopted in this study for both irrigants was based on a previous study ${ }^{7}$ that observed positive antimicrobial and detoxifying effects of QMiX also at 2 minutes. The QMiX contains EDTA, CHX, and cetrimide, a detergent that decreases surface tension and increases wettability and penetrability ${ }^{6}$. However, similar to the results from earlier studies, the QMiX was as effective as $17 \%$ EDTA in debris/smear layer removal ${ }^{6,10,18}$. In turn, Nogo-Zivanovi et al. and Vemuri et al. showed that 


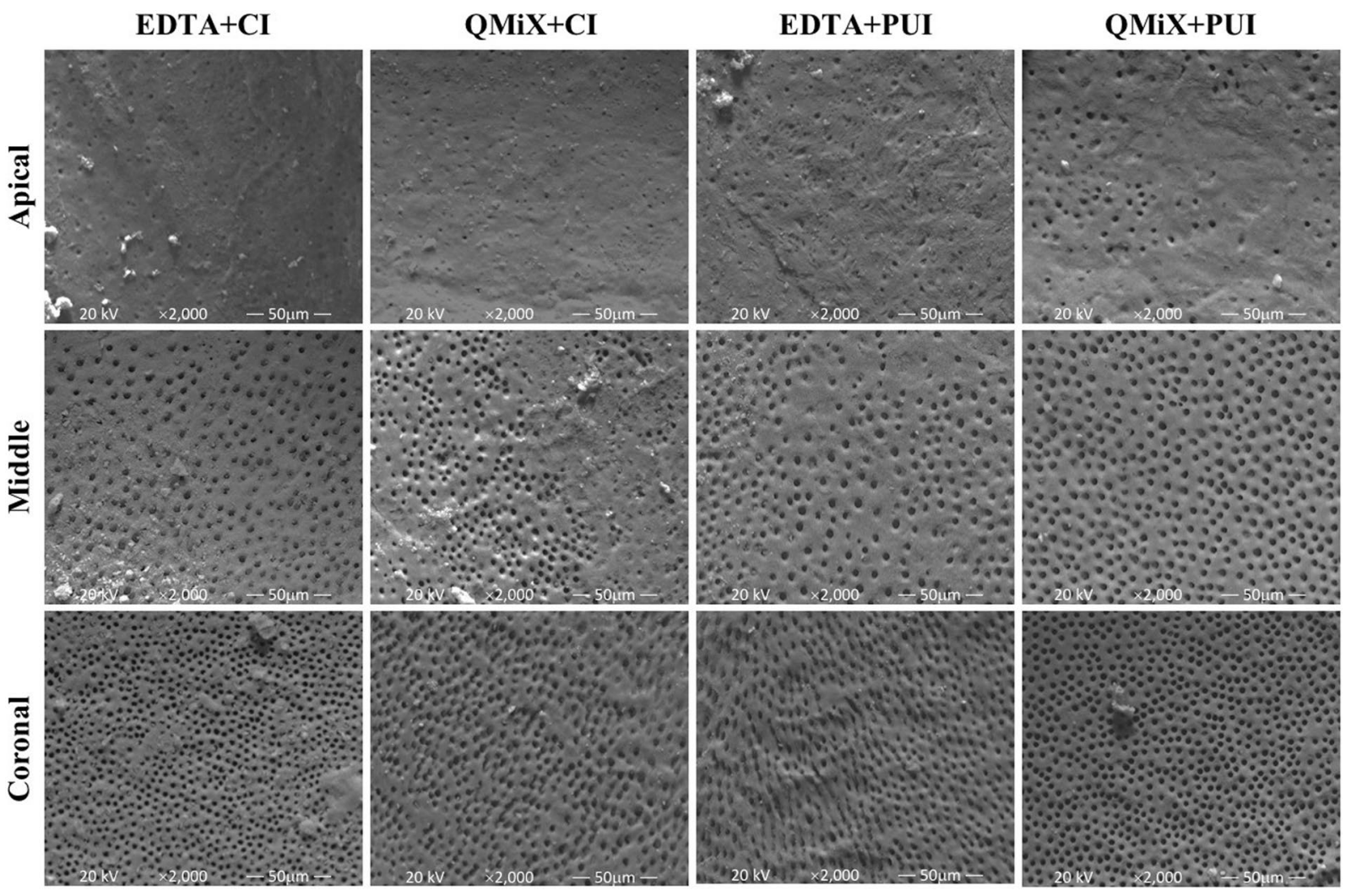

Figure 2. Representative SEM images of debris and smear layer removal from the root canal walls in each group and root third (original magnification, $\times 2,000$ ).

\begin{tabular}{|l|l|l|l|l|}
\hline Groups & Apical third & Middle third & Coronal third & $\boldsymbol{p}$ value \\
\hline EDTA + CI & $684 \pm 175^{\mathrm{aA}}$ & $1391 \pm 164^{\mathrm{aB}}$ & $1927 \pm 303^{\mathrm{aC}}$ & $<0.001$ \\
\hline QMiX + CI & $677 \pm 211^{\mathrm{aA}}$ & $1392 \pm 218^{\mathrm{aB}}$ & $1951 \pm 191^{\mathrm{aC}}$ & $<0.001$ \\
\hline EDTA + PUI & $690 \pm 174^{\mathrm{aA}}$ & $1743 \pm 177^{\mathrm{bB}}$ & $2162 \pm 191^{\mathrm{aC}}$ & $<0.001$ \\
\hline QMiX + PUI & $847 \pm 148^{\mathrm{aA}}$ & $1702 \pm 152^{\mathrm{bB}}$ & $2113 \pm 167^{\mathrm{aC}}$ & $<0.001$ \\
\hline$p$ value & $>0.05$ & $<0.001$ & $>0.05$ & \\
\hline
\end{tabular}

Table 2. Comparison among groups considering the maximum depth of sealer penetration in $\mu \mathrm{m}$ (mean $\pm \mathrm{SD}$ ) in each root canal third. Tukey's test (multiple comparisons): different lowercase letters represent statistically significant difference among groups $(\mathrm{p}<0.05)$ and different capital letters represent statistically significant difference among thirds $(\mathrm{p}<0.05)$. $\mathrm{SD}=$ standard deviation.

\begin{tabular}{|l|l|l|l|l|}
\hline Groups & Apical third & Middle third & Coronal third & $\boldsymbol{p}$ value \\
\hline EDTA + CI & $11.14 \pm 1.22^{\mathrm{aA}}$ & $21.48 \pm 2.97^{\mathrm{aB}}$ & $27.30 \pm 7.21^{\mathrm{aC}}$ & $<0.001$ \\
\hline QMiX + CI & $11.07 \pm 1.01^{\mathrm{aA}}$ & $24.10 \pm 3.42^{\mathrm{aB}}$ & $35.65 \pm 7.08^{\mathrm{bC}}$ & $<0.001$ \\
\hline EDTA + PUI & $14.31 \pm 3.53^{\mathrm{bA}}$ & $24.70 \pm 2.77^{\mathrm{aB}}$ & $33.58 \pm 6.85^{\mathrm{abC}}$ & $<0.001$ \\
\hline QMiX + PUI & $20.85 \pm 2.38^{\mathrm{cA}}$ & $24.60 \pm 2.91^{\mathrm{aA}}$ & $35.03 \pm 5.26^{\mathrm{abB}}$ & $<0.001$ \\
\hline$p$ value & $<0.001$ & $>0.05$ & $<0.05$ & \\
\hline
\end{tabular}

Table 3. Comparison among groups considering the percentage (\%) of sealer penetration (mean $\pm \mathrm{SD})$ in each root canal third. Tukey's test (multiple comparisons): different lowercase letters represent statistically significant difference among groups $(\mathrm{p}<0.05)$ and different capital letters represent statistically significant difference among thirds $(\mathrm{p}<0.05)$. $\mathrm{SD}=$ standard deviation.

QMiX removes the smear layer in the apical third more effectively than $17 \% \mathrm{EDTA}^{8,28}$. Souza et al. revealed that ultrasonic activation of QMiX was significantly more effective in removing the smear layer in the cervical third than ultrasonic activation of $17 \%$ EDTA $^{26}$. Hence, there is still no consensus as to which chelating solution is the 


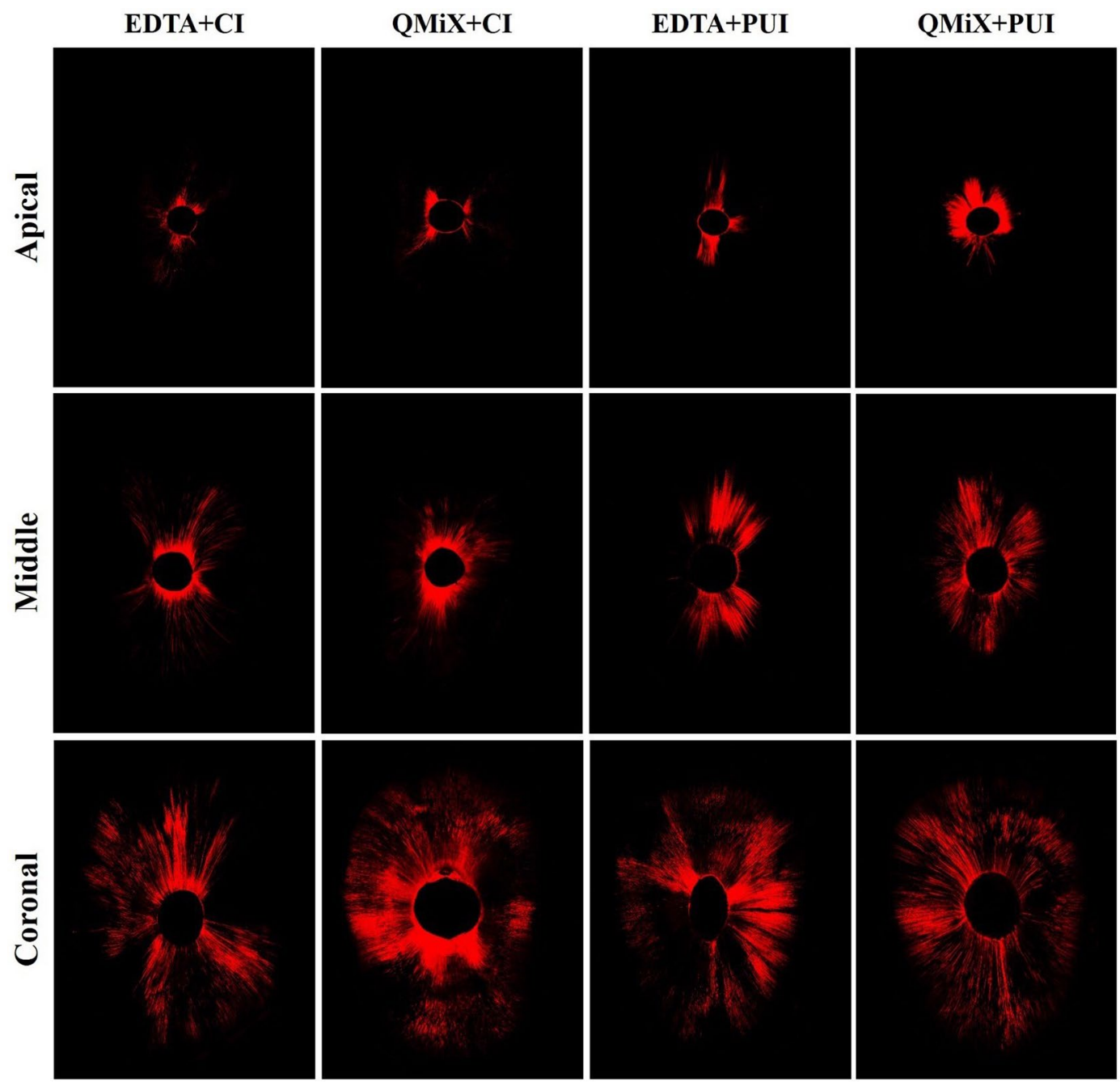

Figure 3. Representative CLSM images of sealer penetration around the root canal walls in each group and root third (original magnification, $\times 10$ ).

most efficient. Differences in experimental design may help to explain the disparate results obtained in the studies mentioned, especially regarding the anatomical particularities of the specimens used, the volume of solution, and time spent for irrigation. Although the protocol recommended is the use of $\mathrm{NaOCl}$ after smear layer removal with EDTA to kill the remaining bacteria, this regimen was not used in this study because $\mathrm{NaOCl}$ after EDTA causes more dentin erosion ${ }^{29}$; also the use of $\mathrm{NaOCl}$ might mask the effect of the solutions tested. Additionally, because the composition of QMiX contains an antimicrobial agent, it exempts the final rinse with $\mathrm{NaOCl}^{6}$.

The effect of final irrigation with QMiX on tubular dentin sealer penetration was compared with 17\% EDTA in previous studies ${ }^{10,30,31}$. However, to our knowledge, this is the first study to report the effects of PUI with QMiX on tubular dentin sealer penetration. The single cone technique was used in this experiment because of its wide use in endodontics and because the filling technique does not interfere with the penetration capacity of sealers ${ }^{32}$. According to our results, PUI improved the maximum depth of sealer penetration in the middle third of the canal when compared to CI, regardless of the chelating solution, which agrees with previous studies using $17 \%$ EDTA $^{15,16}$. The best results for the percentage of sealer penetration were obtained for QMiX + PUI only in the apical third. This may be a direct effect of a better debris/smear layer removal found in the groups treated with PUI in both the middle and apical thirds, confirming the assumption that sealer penetration into the dentinal tubules may be an indicator for the cleaning quality of the canal ${ }^{10}$. Representative SEM images (Fig. 2) showed greater debris/smear layer removal in the groups with the highest percentage of sealer penetration (QMiX + PUI, apical third), with almost all tubules opened and a minimum amount of debris/smear layer attached to the canal walls. The ability of QMiX to promote a higher percentage of sealer penetration may be related to its chemical design containing surfactant (detergent), which increases irrigant flow in the root canal and its contact with the smear layer, improving dentin permeability ${ }^{8}$. In contrast, 17\% EDTA has no detergent in its composition and presents high surface tension and low permeability, which limits its chelating effect ${ }^{33}$. Interestingly, other studies showed that $17 \%$ EDTA and QMiX promoted similar sealer penetration ${ }^{10,30,31}$. 
Overall, the efficacy of the final irrigation protocols for debris/smear layer removal and sealer penetration decreased from the coronal to the apical thirds. This may have been due to two factors. First, the number and diameter of dentinal tubules decrease toward the apical third ${ }^{34}$ and, second, the apical third presents more sclerotic dentin and greater difficulty for irrigant delivery and smear layer removal, which has a direct effect on sealer penetration $^{12,23}$. This result agrees with previous studies that showed that irrigating solutions are less effective in the apical third ${ }^{8,15,18,30,31}$. However, in contrast to CI, no significant difference was observed between the middle and coronal thirds in the debris/smear layer removal for the EDTA + PUI and QMiX + PUI protocols and between the middle and apical thirds in sealer penetration for the QMiX + PUI protocol. This result indicates that both ultrasonic activation and QMiX improved the cleaning and tubular dentin sealer penetration in the middle and apical thirds, respectively. This may be due to the synergistic effect of the ultrasound and the presence of detergent in QMiX that potentially increases its effectiveness by favoring its action on a larger surface area in the root canal and deeper into the dentinal tubules ${ }^{8}$.

The results of this ex vivo study show a close relationship between the final cleaning level of the root canal and tubular dentin sealer penetration. Particularly in the apical third, the QMiX + PUI final irrigating protocol provided an improved debris/smear layer removal of the canal walls and a higher percentage of sealer penetration into the dentinal tubules. This finding is important because the apical third is considered the critical region of the root canal for presenting a greater amount of ramifications of the main root canal. These ramifications are inaccessible to the conventional chemomechanical preparation, which allows harboring remaining bacteria and their by-products and leads to the failure of the endodontic therapy ${ }^{35,36}$. Thus, the present study reveals that final irrigation protocols using PUI and a chelating solution such as QMiX may improve the quality of the cleaning and obturation of the root canal system and it should be considered in the daily clinical practice. However, the level of evidence of the SEM method is limited because only a very small part of the root canal can be evaluated and used to represent the sample ${ }^{14}$. The method of sectioning root dentin samples for analysis in confocal microscopy is already well established as an effective technique that allows determining the presence and extent of penetration of the root canal sealer into the dentinal tubules ${ }^{15,23}$. The major limitation of the present study relates to the laboratory character of the experiment, which restricts extrapolating the results for the clinical practice. Clinical studies are required to validate our results and to evaluate the effect of the final irrigation protocols tested on the clinical success of endodontic treatment.

\section{Conclusion}

Passive ultrasonic irrigation improved debris/smear layer removal and sealer penetration into the dentinal tubules in the apical and middle thirds. When all factors were considered (final irrigating solution, irrigation technique, root canal thirds, debris/smear layer removal, and sealer penetration), the passive ultrasonic irrigation with QMiX showed the best results.

\section{Data availability}

The datasets generated during and/or analyzed during the current study are available from the corresponding author on reasonable request.

Received: 1 August 2019; Accepted: 22 May 2020;

Published online: 25 June 2020

\section{References}

1. Chugal, N. M., Clive, J. M. \& Spångberg, L. S. W. Endodontic infection: some biologic and treatment factors associated with outcome. Oral Surg. Oral Med. Oral Pathol. Oral Radiol. Endod. 96, 81-90 (2003).

2. Buldur, B. \& Kapdan, A. Comparison of the antimicrobial efficacy of the EndoVac system and conventional needle irrigation in primary molar root canals. J. Clin. Pediatr. Dent. 41, 284-288 (2017).

3. Buldur, B., Oznurhan, F. \& Kaptan, A. The effect of different chelating agents on the push-out bond strength of proroot mta and endosequence root repair material. Eur. Oral Res. 53, 88-93 (2019).

4. Violich, D. R. \& Chandler, N. P. The smear layer in endodontics - a review. Int. Endod. J. 43, 2-15 (2010)

5. Zehnder, M. Root canal irrigants. J. Endod. 32, 389-398 (2006).

6. Stojicic, S., Shen, Y., Qian, W., Johnson, B. \& Haapasalo, M. Antibacterial and smear layer removal ability of a novel irrigant, QMiX. Int. Endod. J. 45, 363-371 (2012).

7. Matos, F. S. et al. Effect of EDTA and QMIX ultrasonic activation on the reduction of microorganisms and endotoxins in ex vivo human root canals. Braz. Dent. J. 30, 220-226 (2019).

8. Nogo-Živanović, D., Kanjevac, T., Bjelović, L., Ristić, V. \& Tanasković, I. The effect of final irrigation with MTAD, QMix, and EDTA on smear layer removal and mineral content of root canal dentin. Microsc. Res. Tech. 82, 923-930 (2019).

9. Zhang, R. et al. Antibacterial and residual antimicrobial activities against Enterococcus faecalis biofilm: a comparison between EDTA, chlorhexidine, cetrimide, MTAD and QMix. Sci. Rep. 5, 6-10 (2015).

10. Jardine, A. P. et al. The effect of final irrigation on the penetrability of an epoxy resin-based sealer into dentinal tubules: a confocal microscopy study. Clin. Oral. Investig. 20, 117-123 (2016).

11. Virdee, S. S., Seymour, D. W., Farnell, D., Bhamra, G. \& Bhakta, S. Efficacy of irrigant activation techniques in removing intracanal smear layer and debris from mature permanent teeth: a systematic review and meta-analysis. Int. Endod. J. 51, 605-621 (2018).

12. Munoz, H. R. \& Camacho-Cuadra, K. In vivo efficacy of three different endodontic irrigation systems for irrigant delivery to working length of mesial canals of mandibular molars. J. Endod. 38, 445-448 (2012).

13. Buldur, B. \& Kapdan, A. Comparison of the EndoVac system and conventional needle irrigation on removal of the smear layer in primary molar root canals. Niger. J. Clin. Pract. 20, 1168-74 (2017).

14. Van Der Sluis, L. W. M., Versluis, M., Wu, M. K. \& Wesselink, P. R. Passive ultrasonic irrigation of the root canal: a review of the literature. Int. Endod. J. 40, 415-426 (2007).

15. Akcay, M., Arslan, H., Durmus, N., Mese, M. \& Capar, I. D. Dentinal tubule penetration of AH Plus, iRoot SP, MTA fillapex, and guttaflow bioseal root canal sealers after different final irrigation procedures: a confocal microscopic study. Lasers Surg. Med. 48, 70-76 (2016).

16. Aksel, H., Küçükkaya, E. S., Puralı, N., Serper, A. \& Azim, A. A. Efficacy of different irrigant protocols and application systems on sealer penetration using a stepwise CLSM analysis. Microsc. Res. Tech. 80, 1323-1327 (2017). 
17. Machado, R. et al. Tubular dentin sealer penetration after different final irrigation protocols: a confocal laser scanning microscopy study. Bianchini P, editor. Microsc. Res. Tech. 81, 649-654 (2018).

18. Koçak, S. et al. Influence of passive ultrasonic irrigation on the efficiency of various irrigation solutions in removing smear layer: a scanning electron microscope study. Microsc. Res. Tech. 80, 537-542 (2017).

19. Assouline, L., Fuss, Z., Mazor, Y. \& Weiss, E. Bacterial penetration and proliferation in root canal dentinal tubules after applying dentin adhesives in vitro. J. Endod. 27, 398-400 (2001).

20. Schneider, S. W. A comparison of canal preparations in straight and curved root canals. Oral Surgery, Oral Med. Oral Pathol. 32, 271-275 (1971).

21. Menezes, A. C. S. C., Zanet, C. G. \& Valera, M. C. Smear layer removal capacity of disinfectant solutions used with and without EDTA for the irrigation of canals: a SEM study. Pesqui. Odontol. Bras. 17, 349-355 (2003).

22. Martinho, F. C. et al. Clinical investigation of the efficacy of chemomechanical preparation with rotary nickel-titanium files for removal of endotoxin from primarily infected root canals. J. Endod. 36, 1766-1769 (2010).

23. Ordinola-Zapata, R. et al. Depth and percentage of penetration of endodontic sealers into dentinal tubules after root canal obturation using a lateral compaction technique: a confocal laser scanning microscopy study. Oral Surgery, Oral Med. Oral Pathol. Oral Radiol. Endodontology 108, 450-457 (2009).

24. Blank-Goncalves, L. M., Nabeshima, C. K., Martins, G. H. R. \& Machado, M. E. L. Qualitative analysis of the removal of the smear layer in the apical third of curved roots: conventional irrigation versus activation systems. J. Endod. 37, 1268-1271 (2011).

25. Prado, M. C., Leal, F., Simão, R. A., Gusman, H. \& Prado, M. The use of auxiliary devices during irrigation to increase the cleaning ability of a chelating agent. Restor. Dent. Endod. 42, 105-110 (2017).

26. Souza, M. A. et al. Influence of ultrasonic activation using different final irrigants on antimicrobial activity, smear layer removal and bond strength of filling material. Aust. Endod. J. 45, 209-215 (2019).

27. Desai, P. \& Himel, V. Comparative safety of various intracanal irrigation systems. J. Endod. 35, 545-549 (2009).

28. Vemuri, S. et al. Effect of different final irrigating solutions on smear layer removal in apical third of root canal: a scanning electron microscope study. J. Conserv. Dent. 19, 87-90 (2016)

29. Niu, W., Yoshioka, T., Kobayashi, C. \& Suda, H. A scanning electron microscopic study of dentinal erosion by final irrigation with EDTA and $\mathrm{NaOCl}$ solutions. Int. Endod. J. 35, 934-939 (2002).

30. Aydın, Z. U., Özyürek, T., Keskin, B. \& Baran, T. Effect of chitosan nanoparticle, QMix, and EDTA on TotalFill BC sealers' dentinal tubule penetration: a confocal laser scanning microscopy study. Odontology 107, 64-71 (2019).

31. Tuncer, A. K. Effect of QMix 2in1 on sealer penetration into the dentinal tubules. J. Endod. 41, 257-260 (2015).

32. Kok, D. et al. Evaluation of epoxy resin sealer after three root canal filling techniques by confocal laser scanning microscopy. Microsc. Res. Tech. 75, 1277-1280 (2012).

33. Giardino, L., Ambu, E., Becce, C., Rimondini, L. \& Morra, M. Surface tension comparison of four common root canal irrigants and two new irrigants containing antibiotic. J. Endod. 32, 1091-1093 (2006).

34. Carrigan, P. J., Morse, D. R., Furst, M. L. \& Sinai, I. H. A scanning electron microscopic evaluation of human dentinal tubules according to age and location. J. Endod. 10, 359-363 (1984).

35. Siqueira, J. F. et al. What happens to unprepared root canal walls: a correlative analysis using micro-computed tomography and histology/scanning electron microscopy. Int. Endod. J. 51, 501-508 (2018).

36. Vertucci, F. J. Root canal anatomy of the human permanent teeth. Oral Surgery, Oral Med. Oral Pathol. 58, 589-599 (1984).

\section{Acknowledgements}

The authors would like to thank FAPITEC/SE (Foundation for Support on Research and Technological Innovation of the State of Sergipe) and CAPES (Coordination for the Improvement of Higher Education Personnel - Brazil) for funding the execution of this work through the PROMOB (Academic Mobility Program) - Finance Code 1780/2014. This study was also financed in part by CAPES - Finance Code 001. We are also thankful for the support of CNPq (Council for Scientific and Technological Development - Brazil).

\section{Author contributions}

Felipe de Souza Matos executed the experiments, analyzed and interpreted the data, and wrote the manuscript. Fabrício Rutz da Silva contributed in the realization of the experimental procedures and data collection. Luiz Renato Paranhos and Camilla Christian Gomes Moura provided support for experimental design and reviewed the manuscript. Eduardo Bresciani performed the statistical analysis. Marcia Carneiro Valera designed the study and reviewed the manuscript. All authors have read and approved the final version of the manuscript.

\section{Competing interests}

The authors declare no competing interests.

\section{Additional information}

Correspondence and requests for materials should be addressed to F.d.S.M.

Reprints and permissions information is available at www.nature.com/reprints.

Publisher's note Springer Nature remains neutral with regard to jurisdictional claims in published maps and institutional affiliations.

Open Access This article is licensed under a Creative Commons Attribution 4.0 International License, which permits use, sharing, adaptation, distribution and reproduction in any medium or format, as long as you give appropriate credit to the original author(s) and the source, provide a link to the Creative Commons license, and indicate if changes were made. The images or other third party material in this article are included in the article's Creative Commons license, unless indicated otherwise in a credit line to the material. If material is not included in the article's Creative Commons license and your intended use is not permitted by statutory regulation or exceeds the permitted use, you will need to obtain permission directly from the copyright holder. To view a copy of this license, visit http://creativecommons.org/licenses/by/4.0/.

(C) The Author(s) 2020 